\title{
Pulmonary Hemorrhaging Caused by Acute Severe Mitral Regurgitation during Transcatheter Aortic Valve Implantation
}

\author{
Ryosuke Higuchi ${ }^{1}$, Tetsuya Tobaru ${ }^{1}$, Mike Saji ${ }^{1}$, Keitaro Mahara ${ }^{1}$, Itaru Takamisawa ${ }^{1}$, \\ Jun Shimizu ${ }^{2}$, Shuichiro Takanashi ${ }^{3}$ and Morimasa Takayama ${ }^{1}$
}

\begin{abstract}
:
Transcatheter aortic valve implantation (TAVI) has been validated as a reliable therapy for aortic stenosis (AS), similar to surgical aortic valve replacement. Due to the methodological differences between the two therapeutic options, each has unique complications. We experienced a hitherto unreported complication of TAVI. An 81-year-old man underwent TAVI for severe AS. Acute mitral regurgitation (MR) occurred during valve deployment. Interference of the guidewire with the mitral subvalvular structure caused transient severe MR, leading to the development of pulmonary hemorrhaging. During TAVI, careful attention should be paid to the position of the guidewire, changes in hemodynamics and degree of MR.
\end{abstract}

Key words: pulmonary hemorrhage, lung hemorrhage, transcatheter aortic valve implantation, complication, mitral regurgitation

(Intern Med 57: 1115-1117, 2018)

(DOI: 10.2169/internalmedicine.9712-17)

\section{Introduction}

Surgical aortic valve replacement and transcatheter aortic valve implantation (TAVI) have been established as reliable therapies for severe aortic stenosis (AS). Because of the great methodological differences between the two procedures, some complications are common to both while other complications are specific to each procedure $(1,2)$. TAVIspecific complications include coronary obstruction, aortic root rupture, valve embolization, left ventricle rupture and iliac artery injury.

We report a hitherto unreported complication of bilateral pulmonary hemorrhaging caused by acute severe mitral regurgitation (MR) during TAVI.

\section{Case Report}

An 81-year-old male was admitted with severe AS due to bicuspid aortic valve together with severe right coronary artery stenosis (aortic valve area: $0.70 \mathrm{~cm}^{2}$, peak aortic valve flow: $5.3 \mathrm{~m} / \mathrm{s}$, mean valvular pressure gradient: $60 \mathrm{mmHg}$ ).
He also had systemic sclerosis, Sjogren syndrome, interstitial pneumonitis necessitating oral corticosteroids and home oxygen therapy, chronic renal failure, carotid artery stenosis, chronic pulmonary obstructive disease and previous pulmonary thromboembolism. The calculated Society of Thoracic Surgeons score for surgical aortic valve replacement was 15.3\%. Because of his high surgical risk and moderate frailty, we planned transfemoral TAVI using CoreValve prosthesis (Medtronic, Minneapolis, USA) under local anesthesia to avoid exacerbation interstitial pneumonitis, under transthoracic echocardiography (TTE) guidance. To perform percutaneous coronary intervention (PCI) concurrently with TAVI, clopidogrel was added to warfarin, which had been taken for the secondary prevention of venous thromboembolism. The international normalized ratio of prothrombin time was controlled to 1.75 before the procedure for possible bleeding.

First, using as little contrast medium possible, intervention to the proximal right coronary artery was performed without complication. During preparatory valvuloplasty, TTE showed slight aggravation of MR (Fig. 1A, B, E and F). We attempted to deploy a 29 -mm CoreValve without rapid ven-

${ }^{1}$ Department of Cardiology, Sakakibara Heart Institute, Japan, ${ }^{2}$ Department of Anesthesiology, Sakakibara Heart Institute, Japan and ${ }^{3}$ Department of Cardiovascular Surgery, Sakakibara Heart Institute, Japan

Received: June 24, 2017; Accepted: August 22, 2017; Advance Publication by J-STAGE: December 21, 2017

Correspondence to Dr. Ryosuke Higuchi, rhiguchi@shi.heart.or.jp 

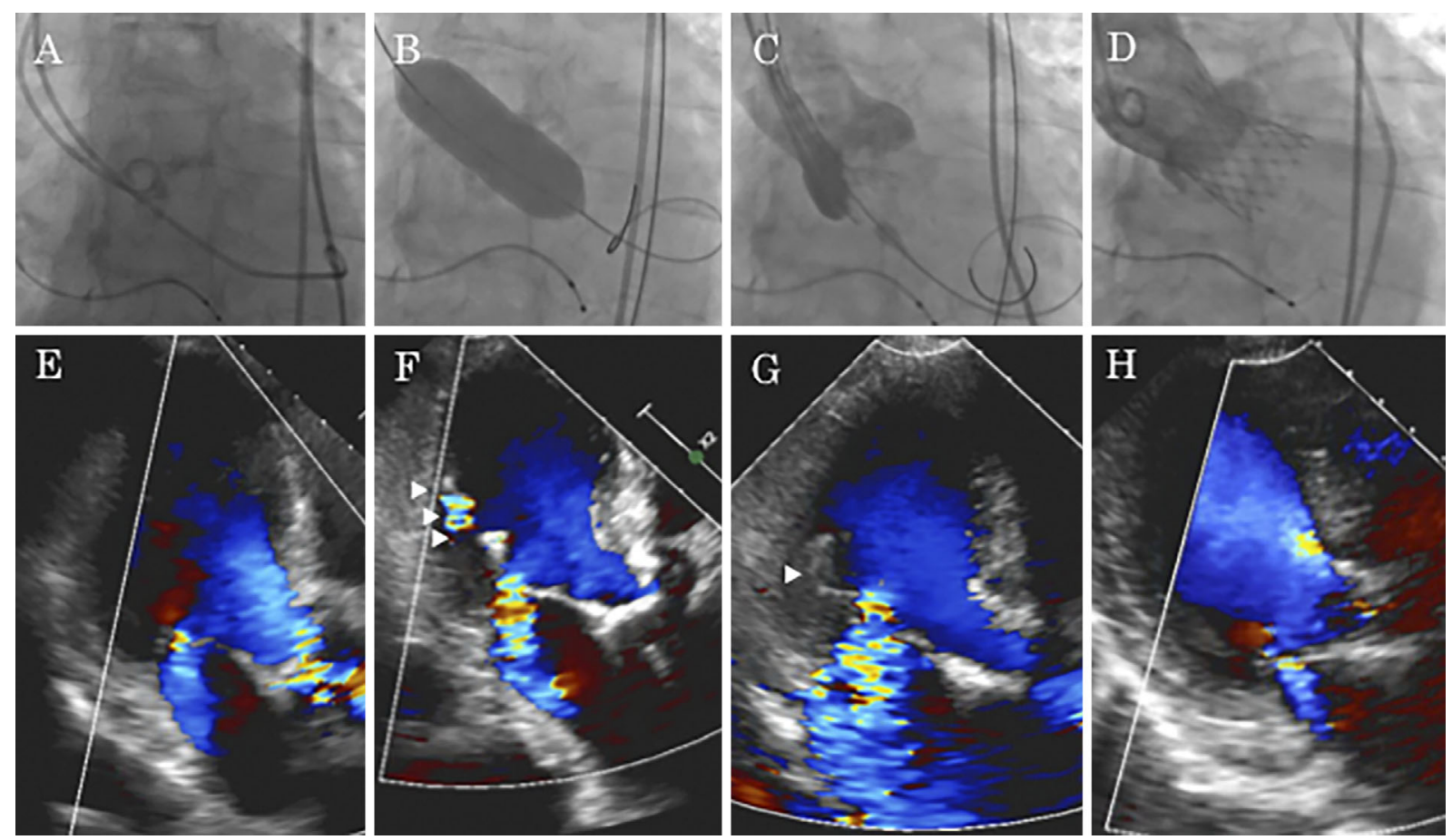

Figure 1. Procedural steps of transcatheter aortic valve implantation and dynamic changes in mitral regurgitation (MR). A: Measurement of the pressure gradient between the ascending aorta and left ventricle. B: Balloon aortic valvuloplasty. C: Expanding the CoreValve prosthesis. D: Final aortography after valve deployment. E: Trivial MR before valvuloplasty. F: Slightly aggravated MR at valvuloplasty (guidewire shown by arrowheads). G: Severe MR during valve deployment (guidewire shown by arrowheads). H: Regressed MR following valve deployment.

tricular pacing. When the prosthetic valve started to expand (Fig. 1C), the systolic blood pressure dropped markedly to 40-50 mmHg. TTE revealed severe MR (Fig. 1G), and blood was blown out via the nasal airway. The CoreValve was deployed smoothly at an acceptable position, with mild perivalvular leakage. Adjusting the position of the left ventricular guidewire (Lunderquist Extra-Stiff Wire Guide; Cook Medical Japan, Tokyo, Japan) stabilized hemodynamics, and resulted in a reduction in MR (Fig. 1D, H). During hemostasis of the common femoral artery, bleeding from the nasal and oral cavity persisted. Both interventions were performed under a heparinized condition. We neutralized unfractionated heparin with protamine sulfate (activated clotting time: maximum $336 \mathrm{~s}, 118 \mathrm{~s}$ after neutralization).

The hemoglobin level decreased from 11.6 to $8.1 \mathrm{~g} / \mathrm{dL}$ on the day after the procedure. Computed tomography demonstrated bilateral pleural hematoma and left lung hemorrhaging (Fig. 2A, B and D-F). Although pulmonary hemorrhaging continued to expand, we managed to stop the bleeding by administering vitamin $\mathrm{K}$, fresh-frozen plasma and hemostatic drug. Respiratory failure was managed by non-invasive positive pressure ventilation. Pulmonary hemorrhaging gradually decreased in size (Fig. 2C), and we continued the administration of a single antithrombotic with clopidogrel. His lung recovered its oxygenation capability, and the patient was discharged home on the 48th postprocedural day.

\section{Discussion}

We experienced a case of bilateral pulmonary hemorrhaging caused by acute severe MR during TAVI. During deployment of the CoreValve, interference of the stiff guidewire with the mitral subvalvular structure caused transient severe MR. The elevated intra-cardiac pressure was transmitted from the left ventricle to the left atrium, pulmonary veins, lungs and pleural space, causing bilateral pulmonary hemorrhaging.

Aguilera et al. (3) reported significant MR during TAVI. They documented occurrence or aggravation of significant MR on transesophageal echocardiography in 11 of 129 cases $(8.5 \%)$. The causes of MR included aortic prosthesis impingement, systolic anterior movement of the anterior mitral leaflet, commissural tearing of the valve and functional mechanism. All significant MR regressed to grade II or less at discharge, and none of the cases required mitral valve surgery. In our case, slight aggravation of MR was observed as the stiff guidewire was inserted, suggesting interference of the guidewire with the subvalvular structure. Stress to the guidewire generated by valve deployment led to transient severe MR. Injury of the mitral valve during TAVI has been reported $(4,5)$. In our case, MR regressed after valve deployment, suggesting no organic damage to the mitral valve. Although transesophageal echocardiography is generally superior to transthoracic echocardiography for continuous 

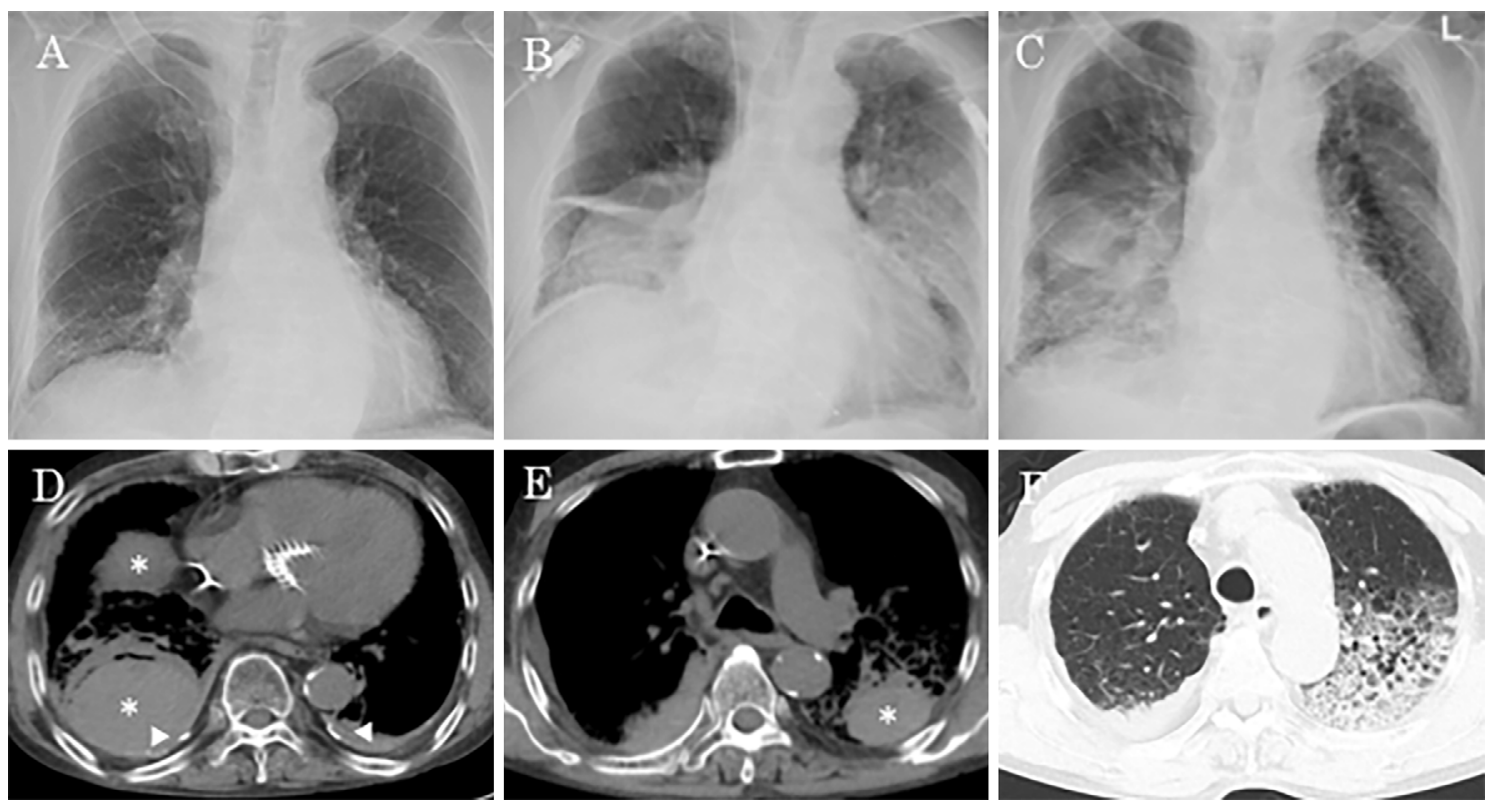

Figure 2. Bilateral pulmonary hemorrhaging on chest X-ray and computed tomography. Chest Xray before transcatheter aortic valve implantation (TAVI) (A), on the day after TAVI (B) and at discharge (C). D-F: Computed tomography on the day after TAVI showing pleural hematoma (asterisk), calcified pleura (arrowheads) and lung hemorrhaging.

monitoring and assessment of MR etiology, we used transthoracic echocardiography, as local anesthesia was used for the procedure. Particularly when deploying a transcatheter heart valve, the position of the guidewire, hemodynamic status and degree of MR must be monitored carefully.

Our patient was prone to bleeding because he was taking clopidogrel and warfarin. He had simple and discrete right coronary artery stenosis eligible for PCI, and we chose concurrent PCI performed with TAVI in light of his hemodynamic stability. Because he had been at risk for recurrence of venous thromboembolism during the peri-procedural period, we decided to maintain warfarin at a therapeutic dose, and added clopidogrel to prevent early stent thrombosis after PCI. Warfarin itself carries a possible side effect of pulmonary hemorrhaging, so it may have promoted this unusual complication. Furthermore, his lung and vascular tissues were probably fragile due to chronic corticosteroids therapy. Acute MR transmitted the elevated intra-cardiac pressure retrogradely from the left ventricle to the left atrium, pulmonary veins, lungs and pleural space. This resulted in bilateral pulmonary hemorrhaging. Computed tomography showed calcified pleura suggestive of previous pleuritis, implying the possible existence of abnormal blood vessels that were susceptible to elevated pressure.

We experienced a case of bilateral pulmonary hemorrhaging caused by acute severe MR during TAVI. Careful attention should be paid during TAVI to the position of the guidewire, changes in hemodynamics and degree of MR.
Author's disclosure of potential Conflicts of Interest (COI).

Tetsuya Tobaru: Advisory role, Edwards Lifesciences and Medtronic.

\section{Acknowledgement}

The authors would like to thank the other members of the TAVI team: Kenichi Hagiya, MD; Yuko Utanohara, MD; Kazuhiro Naito, MD and Nobuo Iguchi, MD.

\section{References}

1. Smith CR, Leon MB, Mack MJ, et al. Transcatheter versus surgical aortic-valve replacement in high risk patients. N Engl J Med 364: 2187-2198, 2011.

2. Adams DH, Popma JJ, Reardon MJ, et al. Transcatheter aorticvalve replacement with a self-expanding prosthesis. N Engl J Med 370: 1790-1798, 2014.

3. López-Aguilera J, Mesa-Rubio D, Ruiz-Ortiz M, et al. Mitral regurgitation during transcatheter aortic valve implantation: the same complication with a different mechanism. J Invasive Cardiol 26: 603-608, 2014.

4. Cozzarin A, Cianciulli TF, Guidoin R, et al. CoreValve prosthesis causes anterior mitral leaflet perforation resulting in severe mitral regurgitation. Can J Cardiol 30: 1108.e11-1108.e13, 2014.

5. Raschpichler M, Seeburger J, Strasser RH, Misfeld M. Corevalve prosthesis causes anterior mitral leaflet perforation resulting in severe mitral regurgitation and subsequent endocarditis. Eur Heart J 35: $1587,2014$.

The Internal Medicine is an Open Access article distributed under the Creative Commons Attribution-NonCommercial-NoDerivatives 4.0 International License. To view the details of this license, please visit (https://creativecommons.org/licenses/ by-nc-nd/4.0/).

(C) 2018 The Japanese Society of Internal Medicine Intern Med 57: 1115-1117, 2018 